\title{
Motivation for Adopting Emergency Response Technology in Community Settings
}

\author{
Philip Fei Wu \\ College of Information Studies \\ University of Maryland \\ College Park, MD, U.S.A. \\ $+13016054790$ \\ fwu@umd.edu
}

\begin{abstract}
My dissertation work focuses on the motivation for adopting emergency response technology in community settings. The main purpose of my study is to understand various factors that affect community members' motivation and ability to participate in ICT-enabled emergency response. The key questions my research intends to answer are: What motivates community members to adopt and use emergency response technologies? What prevents them from using such technologies? What can be done to lower the cognitive, social, and technical barriers of adopting emergency response systems? Answers to these questions not only will inform the system design and assist practitioners in deploying and promoting response systems, but will also provide useful insights to researchers interested in how technologies can facilitate communication and cooperation among community members, especially in response to high-stress, high-stakes situations.
\end{abstract}

\section{Categories and Subject Descriptors}

H.1.2 [MODELS AND PRINCIPLES]: User/Machine Systems - Software psychology.

\section{General Terms}

Design, Human Factors, Theory

\section{Keywords}

Motivation, emergency response, technology adoption, community

\section{INTRODUCTION}

Pervasive information and communication technologies (ICTs) have transformed the landscape of community response to emergencies. Some recent large-scale emergencies (e.g., 2007 Southern California Wildfires) have exemplified the potential power of ICTs in facilitating community emergency response in many ways [1]. While attention to community-based emergency response is growing in the literature, we still lack a systematic understanding of use of response technologies by community members and of differences in adoption of these

(c) The Author 2008 .

Published by the British Computer Society technologies. One central issue that remains understudied is individuals' motivations for adopting and using response technologies. We have learned from many past experiences that 'build it and they will come' is a false assumption. We need to answer some fundamental questions such as: What factors motivate community members to adopt an emergency response system? What factors prevent them from using such systems? And what can be done to lower barriers to adoption and to promote active use?

\section{RESERCH PLAN \& METHODS}

In seeking to answer these research questions, my dissertation draws upon theoretical perspectives from social psychology, emergency response, and technology acceptance research to explore the motivation for using community response technology along three dimensions: cognitive (individual), social (community), and technical (technology). I examine different motivational factors in each dimension and propose an integrated research model to study the phenomenon (Figure 1).

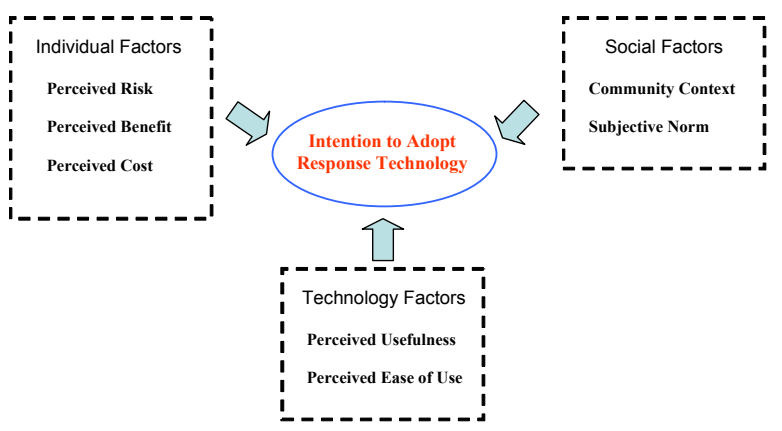

Figure 1. Integrated Research Model

The response technology system that I use as a central case in my study is UMD Alerts - an SMS-based emergency alert system that is currently in use at the University of Maryland. The University has put great effort into promoting the system to the community using various marketing strategies since it was launch in April 2007, but student subscriptions were still rather low - only about $20 \%$ of the student population signed up. In fact, the University of Maryland is by no means a special case. USA Today reported that at many of the schools with the SMS alert services, students are "slow to embrace text alerts" [2].

I use a three-phase, mixed-methods approach to investigate the motivational factors illustrated in the research model:

Phase 1: In-depth interviews and focus groups to explore students' perceptions, attitudes, and opinions regarding UMD Alerts and to identify factors that may affect subscription to the service. The qualitative interview data will broaden and enrich 
the theoretical model in Figure 1, and will increase the internal validity of quantitative data to be collected in Phase 2 .

Phase 2: A quantitative survey questionnaire to gather data about students' motivations for subscribing (or not subscribing) to UMD Alerts, as well as information related to individual, social, and technology factors. The survey data will be analyzed using statistical tools to identify associations between motivational factors and the intention/action of using UMD Alerts.

Phase 3: Interviews and focus groups to make sense of the survey results and to gain a "thick description" of the sociotechnical context of the system adoption.

The results from these three empirical studies will be triangulated and integrated along the way of data analyses and discussions.

\section{INITIAL FINDINGS}

The Phase 1 of this study has been completed, which involved 9 individual in-depth interviews and one focus group (5 participants). The results of the interviews and the focus group indicate that for an emergency response system the characteristics of the information being transferred through the system are critical in determining the perceived usefulness. Some characteristics of information are tied to the technology itself (e.g. accessibility), whereas others have to do with the content of the message being transferred (e.g., relevancy). While all interviewees admitted that UMD Alerts is "useful" because it allows "anywhere anytime" access, many had concerns regarding the irrelevancy of messages to their personal needs. Currently, UMD Alerts disseminates the same information to all subscribers, and the relevancy of the information is determined by the system administrators (i.e., the University police and officials). However, students seem to have different viewpoints with regard to what is "relevant" emergency information to them.

Sending irrelevant text messages to students, who are often overloaded with digital information from all kinds of other sources, created a perception of information overload. The issue of information overload is intensified by some contextual factors of this particular university community. The relevancy of information and the amount of information as perceived by students are heavily influenced by their experiences with the existing emergency communication system and the local environment. Participants showed very low expectation on community safety as the University is located in a metropolitan area with high crime rate. In addition, since the University police also send after-the-fact crime reports to students' university email inboxes - sometimes multiple emails per week, students are being "desensitized" by the "flood" of the "useless reports."

On the other hand, since most college students nowadays are fluent with SMS, it was expected that all the participants said that use of UMD Alerts was "easy." The only issue that falls into the territory of "ease of use," which is closely related to information relevancy and information overload, is the controllability of receiving alert messages. In this context, controllability includes both the ability to customize the types of messages to be received and the flexibility to modify the system behaviors.

Based on these findings and other themes observed in the interviews and the focus group, I refined my research model as following (Figure 2):

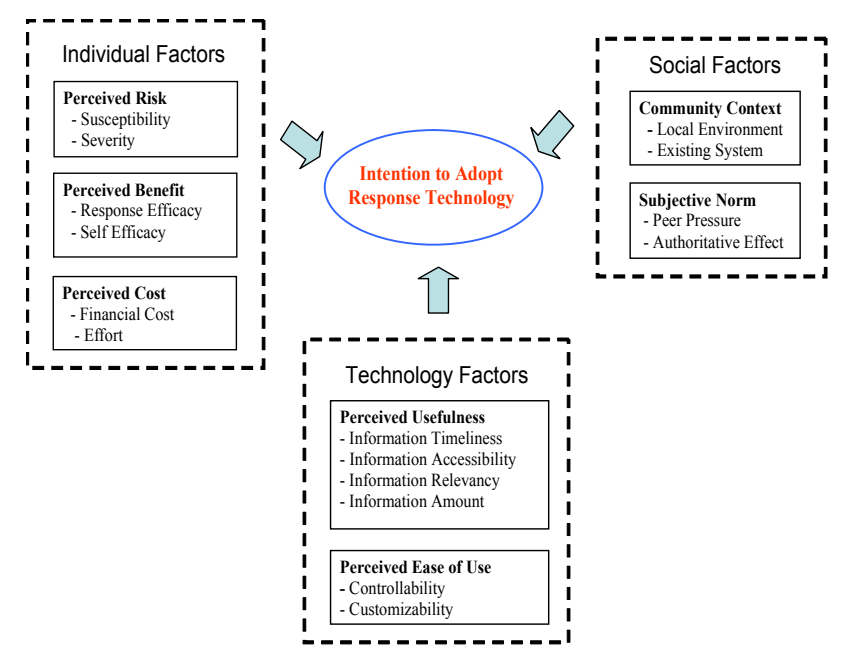

Figure 2. Refined Research Model

The survey questionnaire, which is based on the refined model, intends to quantitatively examine the relationships among these factors in the model, as well as the associations between the factors and the intention of adoption. The questionnaire has been released to the University of Maryland students in the Web survey format. At the time of this writing, 275 responses have been collected. Analyses of the data will be conducted soon. If this paper is accepted, I will be able to report the survey results at the Consortium.

\section{TENTATIVE CONCLUSIONS}

From analyzing the qualitative data, it became clear that motivation for response technology adoption is a complex construct that is influenced by factors specific to the system and the context in which the system is situated. Furthermore, these different types of factors are interacting with one another. As Kling [3] points out, the use of information and communication technologies occurs in a much more complex social environment where social and technological elements intertwined together, forming "socio-technical interaction networks." The use of UMD Alerts, a seemingly straightforward communication technology, is no exception.

\section{ACKNOWLEDGMENTS}

I would like to thank Professor Jennifer Preece and Dr. Yan Qu for advising me on this work.

\section{REFERENCES}

[1] Sutton, J., Palen, L., and Shklovski, I. Backchannels on the front lines: Emergent uses of social media in the 2007 southern California wildfires. In Proceedings of the 5th International Conference on Information Systems for Crisis Response and Management (ISCRAM 2008) (Washington, DC, May 4-7, 2008).

[2] Zagier, A. S. College students slow to embrace text alerts. USA Today (2008, February 28).

[3] Kling, R. Learning about information technologies and social change: The contribution of social informatics. The Information Society, 16, 3 (July 2000), 217-232. 